\title{
Association Between Albumin-Bilirubin Score at Admission and In-Hospital Mortality in Patients with Acute Heart Failure
}

\author{
Takayuki Kawata, ${ }^{1}$ MD, Atsushi Ikeda, ${ }^{1}$ MD, Hiroshi Masuda, ${ }^{1}$ MD and Shunsuke Komatsu, ${ }^{1}$ MD
}

\begin{abstract}
Summary
Liver dysfunction is one of the most recognized complications in patients with acute heart failure (HF) and therefore a liver function score may be useful for risk-stratification in those patients. Recently, the albuminbilirubin (ALBI) score was developed as a new model to assess liver function in liver disease. We explored the association between the ALBI score at admission and in-hospital mortality in patients with acute HF.

We enrolled 262 patients (median age, 86 years, 137 males) who were admitted to our hospital for treatment of acute HF. The following data were recorded: vital signs, laboratory data including B-type natriuretic peptide (BNP) level, echocardiographic data at admission, demographic and clinical characteristics, and treatment and prognostic information. The Get With the Guidelines-Heart Failure (GWTG-HF) risk score was calculated as an established risk model for each patient. The primary outcome was all-cause in-hospital mortality.

During hospitalization, 37 patients $(14.1 \%)$ died. The in-hospital mortality rate was significantly higher in patients with ALBI scores $>-2.25$ compared with patients with ALBI scores $\leq-2.25(21.1 \%$ versus $4.5 \%$, respectively; $P=0.0001$ ). Multivariate analysis revealed that the GWTG-HF score (odds ratio [OR] $1.16,95 \%$ confidence interval $[\mathrm{CI}] 1.08-1.25, P<0.0001)$, BNP level (OR 1.0007, 95\% CI 1.0003-1.001, $P=0.0003$ ) and ALBI score (OR 6.0, 95\% CI 1.8-19.6, $P=0.0017$ ) were independently associated with in-hospital mortality.

Our results indicated that the ALBI score was independently associated with in-hospital mortality in patients hospitalized for acute HF.
\end{abstract}

Key words: Liver function marker, Prognosis

(Int Heart J 2021; 62: 829-836)

$\mathrm{H}$ eart failure (HF) is a major cause of death among elderly individuals in many countries. Since Japan and many Western countries have entered an era of an ultra-aging society, HF has become a significant public health problem. It has been reported that each episode of acute HF may lead to dysfunction or injury of end-organs other than the heart, such as the liver, kidneys, brain, or lungs, which interact with each other, leading to subsequent detrimental consequences. ${ }^{1-3)}$ Acute $\mathrm{HF}$ is therefore not a simple failure of a single organ. In particular, liver dysfunction is a well-known major complication in patients with acute HF, and interactions between the heart and the liver have been increasingly recognized.

Recently, the albumin-bilirubin (ALBI) score was developed as a new parameter to assess liver function in patients with liver disease.) The ALBI score incorporates two variables, serum albumin and total bilirubin, and was developed in 2015 in patients with hepatocellular carcinoma ${ }^{4)}$ in response to problems encountered with the Child-Pugh score. These problems were that some factors were subjective (i.e., ascites and encephalopathy), while other factors such as serum albumin and ascites were in- terrelated. Some studies of the usefulness of the ALBI score have reported its ability to predict prognosis and assist in decision making regarding treatment choices for patients with hepatocellular carcinoma, as it is a suitable tool for evaluation of hepatic reserve function., ${ }^{5,6}$

On the other hand, in the area of heart disease, previous reports have shown that both albumin ${ }^{7-9)}$ and bilirubin $^{10,11)}$ alone, are prognostic factors in patients with HF. Moreover, albumin is also a major component of nutritional indicators such as the prognostic nutritional index, geriatric nutritional risk index, and controlled nutritional status. These indicators have attracted attention in recent years as prognostic factors for $\mathrm{HF}^{12,13)}$ Therefore, the ALBI score, as a combined measurement of albumin and total bilirubin, may become a more attractive prognostic index in patients with HF, and not only in patients with hepatocellular carcinoma. However, to date, a study ${ }^{14)}$ which showed an association between ALBI score and one-year mortality after discharge in patients hospitalized for acute HF, remains the only report of ALBI score and HF. In the present study, we hypothesized that the ALBI score at admission is associated with in-hospital mortality in patients hospitalized for acute HF.

From the ${ }^{1}$ Department of Cardiovascular Medicine, Sassa General Hospital, Tokyo, Japan.

Address for correspondence: Takayuki Kawata, MD, Department of Cardiovascular Medicine, Sassa General Hospital, 4-24-15, Tanashicho, Nishi Tokyo, Tokyo 188-0011, Japan. E-mail: dqh07724@nifty.ne.jp

Received for publication February 5, 2021. Revised and accepted April 6, 2021

Released in advance online on J-STAGE July 17, 2021.

doi: 10.1536/ihj.21-080

All rights reserved by the International Heart Journal Association. 


\section{Methods}

Study subjects and protocol: The present study was a single-center, retrospective observational study. Records of patients who were admitted to our hospital for the treatment of acute HF between January 2017 and August 2020 were reviewed, and patients who had appropriate clinical data for analysis were enrolled. Acute HF was defined as rapid-onset $\mathrm{HF}$ or a change in the signs and symptoms of $\mathrm{HF}$ requiring urgent therapy and hospitalization, based on the Framingham criteria. ${ }^{15)}$ Based on these criteria, the clinical diagnosis of acute HF was made by individual cardiologists at our institution. Patients with acute coronary syndrome, acute myocarditis, infective endocarditis, obvious infectious disease, such as respiratory or urinary tract infections, and patients receiving chronic hemodialysis were not included in this study. Vital signs (e.g. blood pressure), laboratory data including B-type natriuretic peptide (BNP) level and echocardiographic data at admission, as well as demographic and clinical characteristics, treatment and prognostic information, were obtained from medical records. The primary outcome was all-cause inhospital mortality. The study protocol was approved by our institutional ethics committee. All study procedures were performed in compliance with the principles outlined in the Declaration of Helsinki.

Comorbidities: The diagnoses of atrial fibrillation, coronary artery disease, valvular heart disease, cardiomyopathy, chronic obstructive pulmonary disease and cerebrovascular accident were assessed by the treating cardiologists. Hypertension was defined as already receiving medical treatment in the present study, since the patients included often received intravenous vasodilator therapy after admission. The following comorbidities were defined at steady state conditions during admission: diabetes was defined as recent use of insulin or antidiabetic drugs, a fasting blood glucose value $\geq 126 \mathrm{mg} / \mathrm{dL}$ and/or a glycosylated hemoglobin value $\geq 6.5 \%$; dyslipidemia was defined as low-density lipoprotein cholesterol $\geq 140 \mathrm{mg} / \mathrm{dL}$ or high-density lipoprotein cholesterol $<40 \mathrm{mg} / \mathrm{dL}$ or triglycerides $\geq 150 \mathrm{mg} / \mathrm{dL}$ or already receiving medical treatment.

Calculation of the ALBI score: Venous blood samples were collected from all patients at admission. The ALBI score was calculated based on the serum albumin and total bilirubin levels using the following formula, as shown in the original report: $\left(\log _{10}\right.$ total bilirubin $[\mathrm{mmol} / \mathrm{L}] \times$ $0.66)+($ albumin $[\mathrm{g} / \mathrm{L}] \times-0.085){ }^{4}{ }^{4}$ The subjects were divided into two groups (ALBI score $\leq-2.25$ and $>$ -2.25 ) based on the mean ALBI score of a previous study that reported the characteristics of the enrolled patients. ${ }^{14)}$ In addition, to investigate the relationship between ALBI grade and in-hospital mortality, patients were classified 3 ALBI grades $(\leq-2.60=$ grade $1,>-2.60$ to $\leq-1.39=$ grade $2,>-1.39=$ grade 3 ) based on the original report. ${ }^{4}$

GWTG-HF risk model: The GWTG-HF risk model that was derived from a very large data set in the United States ${ }^{16)}$ is a useful tool for predicting in-hospital mortality in patients with acute HF. Since the discrimination and calibration of this risk score have also been well validated in Japanese patients with acute $\mathrm{HF},{ }^{17)}$ this score was also calculated as an established prognosticator in the present study for each patient, as originally described. The GWTG-HF risk score is based on race, age, systolic blood pressure, heart rate, blood urea nitrogen, sodium level, and the presence of chronic obstructive pulmonary disease.

Echocardiography: Cardiac chamber quantification by 2D echocardiography was performed according to established guidelines. ${ }^{18}$ Left ventricular (LV) mass was calculated using diastolic measurement of LV diameter and wall thickness according to the cube formula. ${ }^{18)} \mathrm{LV}$ ejection fraction was calculated using the biplane method of disks (modified Simpson's rule). Both mitral inflow velocities and mitral annular motion velocities were recorded in the apical 4-chamber view, and the ratio of peak early diastolic trans-mitral flow velocity to annular velocity (E/e') was calculated as a parameter of LV diastolic function. ${ }^{19)}$ Tricuspid regurgitation velocity and maximal diameter of the inferior vena cava were measured as hemodynamic assessments of the right heart. Our echocardiographic machines and laboratory are maintained under the guidelines of the Japanese Society of Echocardiography. ${ }^{20)}$

Statistical analysis: All data are expressed as the mean \pm standard deviation (SD) for normally distributed variables and as the median with interquartile range (IQR) for nonnormally distributed data. Categorical data are expressed as the number $(\%)$. Group differences were evaluated using the Student $t$ test or Mann-Whitney $U$ test for continuous variables and the chi-square test or Fisher exact test for categorical variables. A correlation analysis was also performed to calculate the Pearson coefficients between the ALBI score and liver function tests. The association of selected variables with in-hospital mortality was assessed by univariate logistic regression analysis, and the variables that were significant in the univariate model were then entered into a multivariate logistic regression analysis. We also calculated the areas under the receiver operating curve to assess the ability of each model to estimate inhospital mortality (Model 1, model based on the GWTGHF risk score; Model 2, model $1+$ BNP level; Model 3, model $2+$ ALBI score). Calculation of continuous net reclassification improvement (NRI) was used to evaluate the differences between the 3 models. A probability value of $<0.05$ was considered significant. All data were analyzed statistically with JMP version 13.0 (SAS Institute, Cary, NC, USA) and R version 4.0.3 (R Foundation for Statistical Computing, Vienna, Austria).

\section{Results}

Patient characteristics: Between January 2017 and August 2020, 289 patients with acute HF were admitted to our hospital, 27 of whom had inadequate clinical data (i.e., lack of BNP level or blood pressure at admission). Therefore, 262 patients were enrolled in the present study.

Table I presents the general characteristics, hemodynamic data at admission, comorbidities, treatment information and mortality data in all patients and patients stratified according to the ALBI score $(\leq-2.25$ and 
Table I. Patient Baseline Characteristics

\begin{tabular}{|c|c|c|c|c|}
\hline Variable & $\begin{array}{c}\text { All } \\
n=262\end{array}$ & $\begin{array}{c}\mathrm{ALBI} \leq-2.25 \\
n=110\end{array}$ & $\begin{array}{c}\mathrm{ALBI}>-2.25 \\
n=152\end{array}$ & $P$ \\
\hline Age, years & $86(79-91)$ & $84(75-91)$ & $87(82-91)$ & 0.046 \\
\hline Male & $137(52.3)$ & $57(51.8)$ & $80(52.6)$ & 0.90 \\
\hline Height, m & $1.55 \pm 0.11$ & $1.56 \pm 0.11$ & $1.55 \pm 0.11$ & 0.71 \\
\hline Weight, kg & $49.1(41.8-58.4)$ & $51.8(44.2-60.7)$ & $46.4(40.7-56.0)$ & 0.0051 \\
\hline BMI, $\mathrm{kg} / \mathrm{m}^{2}$ & $20.2(17.8-23.2)$ & $21.1(18.9-23.7)$ & $19.5(17.5-22.5)$ & 0.0024 \\
\hline NYHA III or IV at admission & $233(88.9)$ & $95(86.4)$ & $138(90.8)$ & 0.32 \\
\hline History of HF hospitalization & $67(25.6)$ & $24(21.8)$ & $43(28.3)$ & 0.25 \\
\hline \multicolumn{5}{|l|}{ Hemodynamic data } \\
\hline Systolic BP, mmHg & $136(120-157)$ & $142(130-160)$ & $130(116-152)$ & 0.0014 \\
\hline Diastolic BP, mmHg & $82(69-96)$ & $84(70-99)$ & $78(66-94)$ & 0.053 \\
\hline Heart rate, bpm & $90(75-111)$ & $91(76-114)$ & $90(73-110)$ & 0.52 \\
\hline \multicolumn{5}{|l|}{ Clinical diagnosis } \\
\hline Atrial fibrillation & $136(51.9)$ & $53(48.2)$ & $83(54.6)$ & 0.32 \\
\hline Hypertension & $151(57.6)$ & $70(63.6)$ & $81(53.3)$ & 0.10 \\
\hline Diabetes & $47(17.9)$ & $21(19.1)$ & $26(17.1)$ & 0.74 \\
\hline Dyslipidemia & $58(22.1)$ & $30(27.3)$ & $28(18.4)$ & 0.099 \\
\hline Coronary artery disease & $64(24.4)$ & $26(23.6)$ & $38(25.0)$ & 0.88 \\
\hline Valvular heart disease & $48(18.3)$ & $21(19.1)$ & $27(17.8)$ & 0.87 \\
\hline Cardiomyopathy & $34(13.0)$ & $13(11.8)$ & $21(13.8)$ & 0.71 \\
\hline Cerebrovascular disease & $32(12.2)$ & $14(12.7)$ & $18(11.8)$ & 0.85 \\
\hline COPD & $26(9.9)$ & $12(10.9)$ & $14(9.2)$ & 0.68 \\
\hline \multicolumn{5}{|l|}{ Medication at admission } \\
\hline Loop diuretic & $142(54.2)$ & $57(51.8)$ & $85(55.9)$ & 0.53 \\
\hline$\beta$ Blocker & $95(36.3)$ & $39(35.5)$ & $56(36.8)$ & 0.89 \\
\hline ACEi/ARB & 109 (41.6) & $48(43.6)$ & $61(40.1)$ & 0.61 \\
\hline MRB & $61(23.3)$ & $21(19.1)$ & $40(26.3)$ & 0.19 \\
\hline \multicolumn{5}{|l|}{ Intravenous treatment } \\
\hline Inotrope & $60(22.9)$ & $18(16.4)$ & $42(27.6)$ & 0.037 \\
\hline Vasodilator & 94 (35.9) & $41(37.3)$ & $53(34.9)$ & 0.70 \\
\hline Diuretic & $242(92.3)$ & $97(88.2)$ & $145(95.4)$ & 0.035 \\
\hline \multicolumn{5}{|l|}{ Respiratory management } \\
\hline NPPV/intubation & $18(6.9)$ & $10(9.1)$ & $8(5.3)$ & 0.32 \\
\hline Length of stay, days & $17(10-24)$ & $15(10-22)$ & $18(11-26)$ & 0.062 \\
\hline In-hospital death & $37(14.1)$ & $5(4.5)$ & $32(21.1)$ & 0.0001 \\
\hline
\end{tabular}

$>-2.25$ ). The median (IQR) age of all patients was 86 (79-91) years. Compared with patients with ALBI scores $\leq-2.25$, patients with ALBI scores $>-2.25$ were significantly older (87 versus 84 years, respectively) and weighed less (46.4 versus $51.8 \mathrm{~kg}$, respectively), and consequently had a lower BMI (19.5 versus $21.1 \mathrm{~kg} / \mathrm{m}^{2}$, respectively). Vital status at admission, percentage of comorbidities, medication at admission, and treatment after admission were similar in both groups, except for systolic blood pressure, intravenous inotrope, and diuretic use. Median (IQR) length of stay was 17 (10-24) days, with no difference between groups. No patients required percutaneous coronary or valve intervention or cardiac surgery to treat or manage HF. During the index hospitalization, 37 patients $(14.1 \%)$ died. The in-hospital mortality rate was significantly higher in patients with ALBI scores $>-2.25$ compared with patients with ALBI scores $\leq$ -2.25 (21.1\% vs. $4.5 \%$, respectively; $P=0.0001$, Figure $1)$. The number of patients stratified according to ALBI grade were as follows: grade $1, n=33$; grade $2, n=220$; grade $3, n=9$. Most of our subjects were classified in ALBI grade 2. Patients who died during hospitalization in each ALBI grade were 0, 32, and 5 patients, respectively. Therefore, in-hospital mortality increased with higher ALBI grades (Figure 1).

Echocardiography and laboratory data: Echocardiographic parameters, laboratory data, and the GWTG$\mathrm{HF}$ risk score at admission are summarized in Table II. The median (IQR) LV ejection fraction of all patients was 45 (35-61) \%. Maximal inferior vena cava diameter was significantly greater in patients with an ALBI score > -2.25 than in those with a score $\leq-2.25$. The other echocardiographic parameters were comparable between the two groups. Regarding laboratory data, in patients with ALBI scores $>-2.25$, hemoglobin and albumin levels were significantly lower, and total bilirubin and BNP levels were significantly higher compared with those of patients with ALBI scores $\leq-2.25$. Liver function tests such as aspartate aminotransferase (AST) and alanine aminotransferase (ALT) were similar in both groups. The 
GWTG-HF risk score was significantly higher in patients with an ALBI score $>-2.25$ (45 versus 42, respectively; $P=0.0045)$. There was no significant correlation between the ALBI score and AST $(r=-0.012, P=0.85)$ or ALT ( $r=0.0091, P=0.88)$, although a weak but significant positive correlation was observed between the ALBI score and total bilirubin ( $r=0.19, P=0.0016)$.

Factors associated with in-hospital mortality: Table III shows the results of the univariate logistic regression analysis of factors associated with in-hospital mortality. Since the GWTG-HF risk score includes age, blood pressure and heart rate, renal function, sodium, and presence

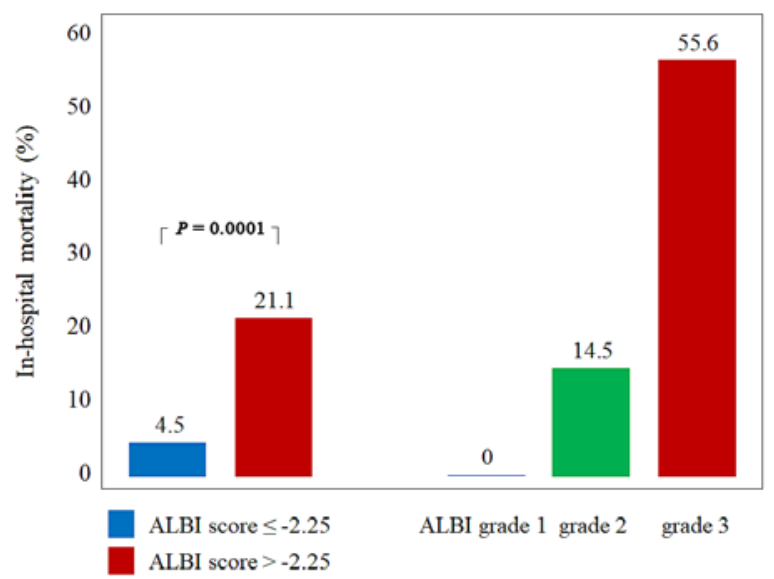

Figure 1. A high ALBI score was associated with a higher mortality. In-hospital mortality was significantly higher in patients with ALBI scores $>-2.25$ compared with that in patients with ALBI scores $\leq-$ 2.25 ( $21.1 \%$ vs. $4.5 \%$, respectively; $P=0.0001)$. In-hospital mortality increased with higher ALBI grades. of chronic obstructive pulmonary disease, these parameters were excluded from this analysis. None of the echocardiographic parameters was associated with outcome except for LV ejection fraction. The higher the LV ejection fraction, the lower the risk of in-hospital death (odds ratio [OR] $0.97,95 \%$ confidence interval [CI] 0.94-0.99, $P=$ 0.0069). In addition, a history of HF hospitalization (OR $2.60,95 \%$ CI $1.26-5.35, P=0.0094)$, serum BNP level (OR 1.0008, 95\% CI 1.0005-1.001, $P<0.0001$ ), GWTGHF risk score (OR 1.20, 95\% CI 1.13-1.28, $P<0.0001)$ and ALBI score (OR 11.6, 95\% CI 4.3-31.2, $P<0.0001$ ) were significantly associated with patient outcomes.

Based on the results of the univariate analysis, a history of HF hospitalization, LV ejection fraction, BNP level, GWTG-HF risk score, and ALBI score were selected as variables for the multivariate analysis. This analysis showed that the BNP level (OR 1.0007, 95\% CI 1.0003-1.001, $P=0.0003$ ), GWTG-HF risk score (OR $1.16,95 \%$ CI $1.08-1.25, P<0.0001)$, and ALBI score (OR 6.0, 95\% CI 1.8-19.6, $P=0.0017$ ) were independently associated with in-hospital mortality (Table IV). Figure 2 and Table $\mathrm{V}$ show the receiver operating curve analysis of each model that estimated in-hospital mortality based on the results of the multivariate analysis. Compared with Model 1, a significant NRI occurred with the addition of the BNP level (Model 2). Compared with Model 2, there was a further significant increase in NRI with the addition of the ALBI score (Model 3).

\section{Discussion}

The key finding of the present study was that a high ALBI score on admission was independently associated with a high in-hospital mortality in patients hospitalized

Table II. Echocardiographic Data, Laboratory Data, GWTG-HF Risk Score and ALBI Score at Admission

\begin{tabular}{lcccc}
\hline Variable & All & ALBI $\leq-2.25$ & ALBI $>-2.25$ & $n=152$ \\
& $n=262$ & $n=110$ & & \\
\hline Echocardiography & & & & \\
LV mass index, g/m ${ }^{2}$ & $121.9 \pm 38.3$ & $120.4 \pm 36.1$ & $123.1 \pm 39.8$ & 0.29 \\
LV ejection fraction, $\%$ & $45(35-61)$ & $46(37-61)$ & $45(33-61)$ & 0.28 \\
E/e' & $21(17-30)$ & $22(17-29)$ & $21(16-31)$ & 0.84 \\
TR velocity, m/second & $2.9 \pm 0.6$ & $2.9 \pm 0.6$ & $2.9 \pm 0.6$ & 0.32 \\
Maximal IVC diameter, mm & $18.8 \pm 4.4$ & $18.0 \pm 4.3$ & $19.3 \pm 4.3$ & 0.021 \\
Laboratory data & & & & \\
Hemoglobin, g/dL & $11.9 \pm 2.4$ & $12.2 \pm 2.2$ & $11.6 \pm 2.5$ & 0.034 \\
Albumin, g/dL & $3.5(3.2-3.8)$ & $3.8(3.6-3.9)$ & $3.2(2.9-3.4)$ & $<0.0001$ \\
Sodium, mEq/L & $139(137-142)$ & $139(136-142)$ & $139(137-142)$ & 0.33 \\
Creatinine, mg/dL & $1.01(0.77-1.41)$ & $1.00(0.77-1.35)$ & $1.03(0.77-1.52)$ & 0.58 \\
eGFR, mL/minute/1.73 m ${ }^{2}$ & $46(32-64)$ & $48(34-62)$ & $45(31-67)$ & 0.54 \\
BUN, mg/dL & $24.5(17.9-38.0)$ & $23.5(16.6-34.8)$ & $25.1(18.2-41.1)$ & 0.24 \\
Total bilirubin, mg/dL & $0.86(0.63-1.24)$ & $0.74(0.59-1.12)$ & $0.95(0.68-1.33)$ & 0.0013 \\
AST, IU/L & $31(23-46)$ & $30(22-46)$ & $32(23-48)$ & 0.47 \\
ALT, IU/L & $21(13-40)$ & $20(13-39)$ & $22(13-42)$ & 0.78 \\
BNP, pg/mL & $732(453-1271)$ & $659(358-926)$ & $841(509-1561)$ & 0.0012 \\
GWTG-HF risk score & $44 \pm 8$ & $42 \pm 8$ & $45 \pm 8$ & 0.0045 \\
ALBI score & $-2.15 \pm 0.40$ & $-2.50 \pm 0.20$ & $-1.89 \pm 0.30$ & $<0.0001$ \\
\hline
\end{tabular}

Data are presented as the mean \pm SD or median (IQR). LV indicates left ventricular; E/e', the ratio of peak early diastolic trans-mitral flow velocity to annular velocity; TR, tricuspid regurgitation; IVC, inferior vena cava; eGFR, estimated glomerular filtration rate; BUN, blood urea nitrogen; AST, aspartate aminotransferase; ALT, alanine aminotransferase; BNP, B-type natriuretic peptide; GWTG-HF, Get With the Guidelines-Heart Failure; and ALBI, albumin-bilirubin. 
Table III. Univariate Logistic Regression Analysis of Factors Associated with Outcome

\begin{tabular}{lcc}
\hline Variable & Odds ratio (95\% CI) & $P$ \\
\hline Male & $0.74(0.37-1.49)$ & 0.41 \\
BMI & $0.96(0.87-1.05)$ & 0.33 \\
NYHA III or IV at admission & $5.1(0.67-38.8)$ & 0.11 \\
History of HF hospitalization & $2.60(1.26-5.35)$ & 0.0094 \\
Atrial fibrillation & $0.86(0.43-1.72)$ & 0.67 \\
Hypertension & $1.24(0.61-2.54)$ & 0.55 \\
Diabetes & $1.32(0.56-3.09)$ & 0.53 \\
Dyslipidemia & $0.80(0.33-1.92)$ & 0.61 \\
Coronary artery disease & $1.85(0.88-3.89)$ & 0.11 \\
Valvular heart disease & $1.82(0.81-4.08)$ & 0.16 \\
Cardiomyopathy & $0.79(0.26-2.38)$ & 0.67 \\
LV mass index & $1.00(0.99-1.01)$ & 0.80 \\
LV ejection fraction & $0.97(0.94-0.99)$ & 0.0069 \\
E/e' & $1.03(0.99-1.06)$ & 0.072 \\
TR velocity & $1.002(0.57-1.78)$ & 0.99 \\
Maximal IVC diameter & $1.06(0.97-1.15)$ & 0.21 \\
Hemoglobin & $0.90(0.77-1.04)$ & 0.15 \\
AST & $1.001(0.99-1.004)$ & 0.19 \\
ALT & $1.002(0.99-1.006)$ & 0.22 \\
BNP & $1.0008(1.0005-1.001)$ & $<0.0001$ \\
GWTG-HF risk score & $1.20(1.13-1.28)$ & $<0.0001$ \\
ALBI score & $11.6(4.3-31.2)$ & $<0.0001$ \\
\hline Abreviations in Tabl I & &
\end{tabular}

Abbreviations as in Tables I and II.

Table IV. Multivariate Logistic Regression Analysis of Factors Associated with Outcome

\begin{tabular}{lcc}
\hline Variable & Odds ratio (95\% CI) & $P$ \\
\hline History of HF hospitalization & $1.0008(0.36-2.77)$ & 0.99 \\
LV ejection fraction & $0.99(0.96-1.03)$ & 0.84 \\
BNP & $1.0007(1.0003-1.001)$ & 0.0003 \\
GWTG-HF risk score & $1.16(1.08-1.25)$ & $<0.0001$ \\
ALBI score & $6.0(1.8-19.6)$ & 0.0017 \\
\hline
\end{tabular}

Abbreviations as in Tables I and II.

for acute HF. Moreover, we confirmed that the GWTG-HF risk score and BNP level, established as prognostic indicators, were strongly associated with in-hospital mortality even in very elderly patients.

Albumin is a hepatic protein and its plasma concentration is controlled by several factors, including the rate of albumin synthesis, albumin distribution, catabolic rate, and exogenous albumin loss. ${ }^{21,22)}$ Synthesis of albumin is affected by nutritional intake, variations in colloid oncotic pressure, and the presence of systemic inflammation. ${ }^{21,23)}$ Plasma albumin levels are known to be decreased in inflammatory conditions, including infection, trauma, and surgery. ${ }^{24)}$ Previous clinical trials demonstrated that the HF syndrome is characterized by activation of inflammatory factors, including C-reactive protein and circulating cytokines and chemokines. ${ }^{25,26)}$ Bonilla-Palomas, et al. $^{27)}$ showed in their detailed study that both malnutrition and inflammatory activation were the most important etiological factors associated with hypoalbuminemia in patients with acute HF. Therefore, we presume that hypoalbuminemia is common in patients with HF, and to date, this condition is a well-established predictor for short-term prognosis in patients with acute $\mathrm{HF}^{7,27)}$ In recent years, there has been increasing evidence that 3 nutritional indices, the prognostic nutritional index, geriatric nutritional risk index, and controlled nutritional status are all associated with the short- and long-term prognosis of patients with acute $\mathrm{HF}{ }^{12,13)}$ with the main component of each nutritional index being albumin. These findings indicate that albumin is an important indicator of malnutrition and inflammation in patients with HF. In addition, the mechanism that causes hypoalbuminemia due to liver dysfunction associated with acute $\mathrm{HF}$ is also possible. Liver dysfunction is one of the most recognized complications in patients with acute HF, with the most common cause being liver congestion indicated by the finding of an elevated central venous pressure (CVP) due to right HF. Drazner, et al. ${ }^{28)}$ examined invasive hemodynamic data in detail from 4,079 patients with HF and found that CVP and pulmonary capillary wedge pressure remained concordant in most patients. Therefore, the most common and typical phenomenon in HF is systemic congestion. Several studies have reported that albumin levels may be associated with systemic congestion in patients with acute HF. BonillaPalomas, et al also reported that a low albumin level was associated with a CVP $>15 \mathrm{mmHg}$ estimated by echocardiography. ${ }^{27)}$ Uthamalingam, et al. showed that the presence of severe tricuspid regurgitation is independently associated with low serum albumin. ${ }^{29)}$ A report from $1976^{30)}$ showed that increased microvascular protein escape is present in HF with increased CVP. It is possible that intestinal congestion favors albumin enteric losses. ${ }^{31)}$ Hypoalbuminemia may therefore also be a surrogate marker of liver dysfunction that reflects an elevated CVP. In fact, the present study showed that the maximal inferior vena cava diameter was significantly greater in patients with an ALBI score $>-2.25$ despite them having a smaller body size. 


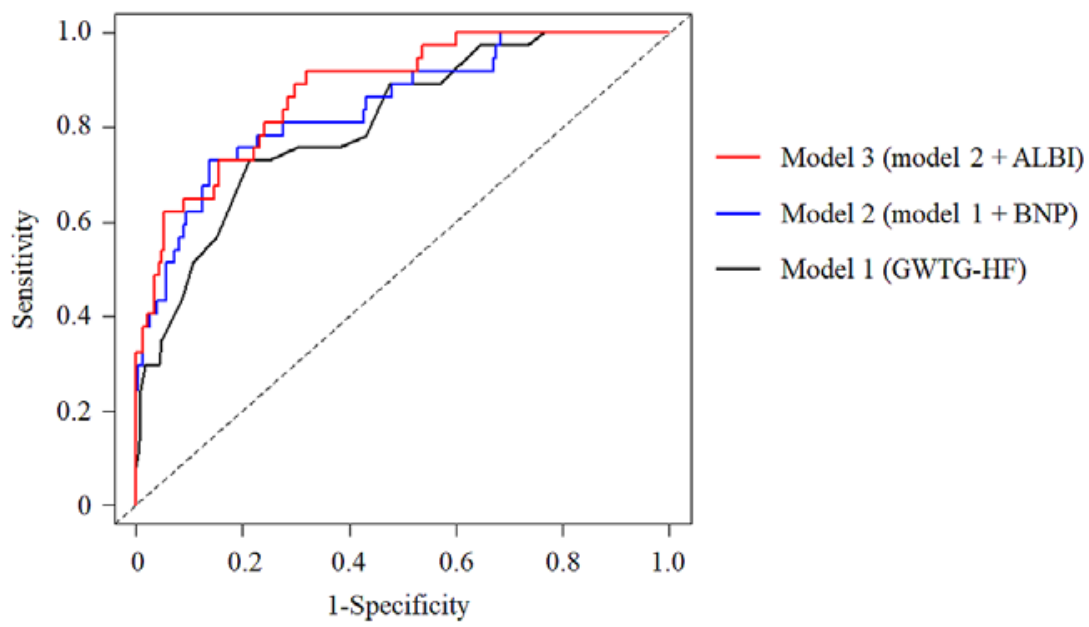

Figure 2. Area under the receiver operating curves for estimating in-hospital mortality in the study patients $(n=262)$. See also Table V.

Table V. Area Under the Curve Analysis of Each Model and Associated Net Reclassification Improvement

\begin{tabular}{lccc}
\hline Variable & AUC $(95 \%$ CI $)$ & NRI (95\% CI) & $P$ \\
\hline Model 1 (GWTG-HF risk score) & $0.81(0.73-0.88)$ & & \\
Model 2 (model 1 + BNP level) & $0.85(0.77-0.92)$ & $0.55(0.21-0.89) *$ & 0.0017 \\
Model 3 (model 2 + ALBI score) & $0.88(0.82-0.94)$ & $0.46(0.12-0.80) * *$ & 0.0085 \\
\hline
\end{tabular}

AUC indicates area under the curve; and NRI, net reclassification improvement; other abbreviations as in Tables I and II.*versus Model 1. **versus Model 2.

This result suggests that patients with an ALBI score > -2.25 may have a higher CVP, although this difference is small and invasive data on this association are lacking.

Regarding bilirubin, low hepatic blood flow due to decreased cardiac output and high CVP may induce atrophy of liver cells and edema of the peripheral area, changes that both lead to hypoxic hepatocellular injury. ${ }^{32-34)}$ Hypoxic hepatocellular injury, in turn, causes impaired uptake of indirect bilirubin from blood and secretion of bilirubin directly into bile, leading to an increase in serum bilirubin level. A high CVP also leads to biliary occlusion and subsequently to an increase in serum bilirubin level. In fact, several studies have shown that serum bilirubin correlated positively with CVP and pulmonary artery wedge pressure, and inversely correlated with the cardiac index, though a rise in total bilirubin was not noticeable. ${ }^{32,35)}$ Vishram-Nielsen, et $a l .^{36)}$ also reported that CVP obtained by an invasive method was associated with both markers of biliary excretion (alkaline phosphatase and bilirubin) and liver synthesis function (international normalization ratio) in hospitalized or out-patients with acute HF. An elevated total bilirubin level was independently associated with worse clinical outcomes in both the CHARM program ${ }^{10)}$ and the ASCEND-HF study. ${ }^{11)}$ More recently, in patients with myocardial infarction complicated with $\mathrm{HF}$ and/or systolic dysfunction, the total bilirubin concentration was shown to be an independent predictor of mortality and may improve risk stratification. ${ }^{37)}$ Therefore, the ALBI score, a combined measurement of both albumin and total bilirubin, might reflect, at least in part, liver congestion due to $\mathrm{HF}$ and subsequent liver dysfunction.

Although the ALBI score was developed originally using data of patients with hepatocellular carcinoma to estimate the degree of liver dysfunction, ${ }^{4)}$ the utility of this score can be generalized to patients with cirrhosis irrespective of the etiology of the underlying liver disease (i.e., without hepatocellular carcinoma). ${ }^{38)}$ Therefore, the ALBI score may become a marker of liver dysfunction even in patients without hepatocellular carcinoma. In clinical practice, calculation of the ALBI score, measurement of the BNP level, as well as all of the variables used to obtain the GWTG-HF risk score, are available within the first 1-2 hours of patient evaluation, and these variables are objective indicators not subject to inter-observer errors. Our results showed that not only the GWTG-HF risk score and the BNP level, but also the ALBI score, can provide useful information for patient triage and treatment decisions in patients hospitalized for acute HF.

Limitations: The present study has several important limitations. First, although the treatment of acute HF was based on the guidelines, it was left to the discretion of each attending cardiologist. This may have had some impact on patient outcomes. In addition, an effect due to the decision of the attending physician may also exist regarding the diagnosis of the etiology of HF. Second, this study was conducted at a single center, and consequently included a limited number of patients. Moreover, this study was a retrospective observational study. Since our hospital is located in the suburbs of metropolitan Tokyo, the pa- 
tient population of our hospital may reflect the patient population of general hospitals in Japan. However, prospective, large-scale studies are necessary to confirm our findings.

\section{Conclusion}

The ALBI score was independently associated with in-hospital mortality in patients hospitalized for acute HF. Moreover, as with previous reports, the GWTG-HF risk score and the BNP level also strongly provided short-term prognostic information in patients hospitalized for acute HF.

\section{Disclosure}

Conflicts of interest: The authors declare that there are no relationships to report with any industry.

\section{References}

1. Harjola VP, Mullens W, Banaszewski M, et al. A review on behalf of the Acute Heart Failure Committee of the Heart Failure Association (HFA) of the European Society of Cardiology (ESC). Eur J Heart Fail 2017; 19: 821-36.

2. Smith GL, Lichtman JH, Bracken MB, et al. Renal impairment and outcomes in heart failure: systematic review and metaanalysis. J Am Coll Cardiol 2006; 47: 1987-96.

3. Biegus J, Hillege HL, Postmus D, et al. Abnormal liver function tests in acute heart failure: relationship with clinical characteristics and outcome in the PROTECT study. Eur J Heart Fail 2016; 18: 830-9.

4. Johnson PJ, Berhane S, Kagebayashi C, et al. Assessment of liver function in patients with hepatocellular carcinoma: a new evidence-based approach-the ALBI grade. J Clin Oncol 2015; 33: $550-8$.

5. Hiraoka A, Kumada T, Kudo M, et al. Real-Life Practice Experts for HCC (RELPEC) Study Group and HCC 48 Group (hepatocellular carcinoma experts from 48 clinics). AlbuminBilirubin (ALBI) Grade as part of the Evidence-Based Clinical Practice Guideline for HCC of the Japan Society of Hepatology: a comparison with the Liver Damage and Child-Pugh classifications. Liver Cancer 2017; 6: 204-15.

6. Shao YY, Liu TH, Lee YH, Hsu CH, Cheng AL. Modified CLIP with objective liver reserve assessment retains prognosis prediction for patients with advanced hepatocellular carcinoma. J Gastroenterol Hepatol 2016; 31: 1336-41.

7. Ancion A, Allepaerts S, Oury C, Gori AS, Piérard LA, Lancellotti P. Serum albumin level and hospital mortality in acute nonischemic heart failure. ESC Heart Fail 2017; 4: 138-45.

8. Horwich TB, Kalantar-Zadeh K, MacLellan RW, Fonarow GC. Albumin levels predict survival in patients with systolic heart failure. Am Heart J 2008; 155: 883-9.

9. Suzuki S, Motoki H, Kanzaki Y, et al. A Predictive Model for 6Month Mortality in Elderly Patients with Heart Failure. Int Heart J 2020; 61: 325-31.

10. Allen LA, Felker GM, Pocock S, et al. CHARM Investigators. Liver function abnormalities and outcome in patients with chronic heart failure: data from the Candesartan in Heart Failure: Assessment of Reduction in Mortality and Morbidity (CHARM) program. Eur J Heart Fail 2009; 11: 170-7.

11. Samsky MD, Dunning A, DeVore AD, et al. Liver function tests in patients with acute heart failure and associated outcomes: insights from ASCEND-HF. Eur J Heart Fail 2016; 18: 424-32.

12. Yoshihisa A, Kanno Y, Watanabe S, et al. Impact of nutritional indices on mortality in patients with heart failure. Open Heart
2018; 5: e000730.

13. Nishi I, Seo Y, Hamada-Harimura Y, et al. Ibaraki Cardiovascular Assessment Study-Heart Failure Investigators. Utility of Nutritional Screening in Predicting Short-Term Prognosis of Heart Failure Patients. Int Heart J 2018; 59: 354-60.

14. Matsue Y, Kagiyama N, Yamaguchi T, et al. Clinical and prognostic values of ALBI score in patients with acute heart failure. Heart Lung Circ 2020; 29: 1328-37.

15. Ho KK, Anderson KM, Kannel WB, Grossman W, Levy D. Survival after the onset of congestive heart failure in Framingham Heart Study subjects. Circulation 1993; 88: 107-15.

16. Peterson PN, Rumsfeld JS, Liang L, et al. American Heart Association Get With the Guidelines-Heart Failure Program. A validated risk score for in-hospital mortality in patients with heart failure from the American Heart Association get with the guidelines program. Circ Cardiovasc Qual Outcomes 2010; 3: 25-32.

17. Shiraishi Y, Kohsaka S, Abe T, et al. West Tokyo Heart Failure Registry Investigators. Validation of the Get With The Guideline-Heart Failure risk score in Japanese patients and the potential improvement of its discrimination ability by the inclusion of B-type natriuretic peptide level. Am Heart J 2016; 171: 33-9.

18. Lang RM, Badano LP, Mor-Avi V, et al. Recommendations for cardiac chamber quantification by echocardiography in adults: an update from the American Society of Echocardiography and the European Association of Cardiovascular Imaging. Eur Heart J Cardiovasc Imaging 2015; 16: 233-70.

19. Nagueh SF, Smiseth OA, Appleton CP, et al. Recommendations for the evaluation of left ventricular diastolic function by echocardiography: an update from the American Society of Echocardiography and the European Association of Cardiovascular Imaging. J Am Soc Echocardiogr 2016; 29: 277-314.

20. Nakatani S, Akaishi M, Asanuma T, et al. Guidelines from the Japanese Society of Echocardiography: guidance for the management and maintenance of echocardiography equipment. J Echocardiogr 2015; 13: 1-5.

21. Don BR, Kaysen G. Serum albumin: relationship to inflammation and nutrition. Semin Dial 2004; 17: 432-7.

22. Fuhrman MP, Charney P, Mueller CM. Hepatic proteins and nutrition assessment. J Am Diet Assoc 2004; 104: 1258-64.

23. Mitch WE. Malnutrition: a frequent misdiagnosis for hemodialysis patients. J Clin Invest 2002; 110: 437-9.

24. Gabay C, Kushner I. Acute-phase proteins and other systemic responses to inflammation. N Engl J Med 1999; 340: 448-54.

25. Deswal A, Petersen NJ, Feldman AM, Young JB, White BG, Mann DL. Cytokines and cytokine receptors in advanced heart failure: an analysis of the cytokine database from the Vesnarinone trial (VEST). Circulation 2001; 103: 2055-9.

26. Anand IS, Latini R, Florea VG, et al. Val-HeFT Investigators. $\mathrm{C}$-reactive protein in heart failure: prognostic value and the effect of valsartan. Circulation 2005; 112: 1428-34.

27. Bonilla-Palomas JL, Gámez-López AL, Moreno-Conde M, et al. Hypoalbuminemia in acute heart failure patients: causes and its impact on hospital and long-term mortality. J Card Fail 2014; 20: $350-8$

28. Drazner MH, Brown RN, Kaiser PA, et al. Relationship of rightand left-sided filling pressures in patients with advanced heart failure: a 14-year multi-institutional analysis. J Heart Lung Transplant 2012; 31: 67-72.

29. Uthamalingam S, Kandala J, Daley M, et al. Serum albumin and mortality in acutely decompensated heart failure. Am Heart $\mathrm{J}$ 2010; 160: 1149-55.

30. Hesse B, Parving HH, Lund-Jacobsen H, Noer I. Transcapillary escape rate of albumin and right atrial pressure in chronic congestive heart failure before and after treatment. Circ Res 1976; 39: 358-62.

31. Battin DL, Ali S, Shahbaz AU, Massie JD, Munir A, Davis RC Jr. Hypoalbuminemia and lymphocytopenia in patients with decompensated biventricular failure. Am J Med Sci 2010; 339: 
$31 \mathrm{e} 5$.

32. Kubo SH, Walter BA, John DH, Clark M, Cody RJ. Liver function abnormalities in chronic heart failure. Influence of systemic hemodynamics. Arch Intern Med 1987; 147: 1227-30.

33. Seeto RK, Fenn B, Rockey DC. Ischemic hepatitis: clinical presentation and pathogenesis. Am J Med 2000; 109: 109-13.

34. Giallourakis CC, Rosenberg PM, Friedman LS. The liver in heart failure. Clin Liver Dis 2002; 6: 947-67.

35. Shinagawa H, Inomata T, Koitabashi T, et al. Prognostic significance of increased serum bilirubin levels coincident with cardiac decompensation in chronic heart failure. Circ J 2008; 72 : 364-9.

36. Vishram-Nielsen JKK, Deis T, Balling L, et al. Relationship between invasive hemodynamics and liver function in advanced heart failure. Scand Cardiovasc J 2019; 53: 235-46.

37. Frikha Z, Ferreira JP, Bozec E, et al. High-Risk Myocardial Infarction Database Initiative Investigators. Relation of High Serum Bilirubin to Short-Term Mortality Following a Myocardial Infarction Complicated by Left Ventricular Systolic Dysfunction (from the High-Risk Myocardial Infarction Database Initiative). Am J Cardiol 2018; 121: 1015-20.

38. Oikonomou T, Goulis L, Doumtsis P, Tzoumari T, Abkriviadis E, Cholongitas E. ALBI and PALBI Grades Are Associated with the Outcome of Patients with Stable Decompensated Cirrhosis. Ann Hepatol 2019; 18: 126-36. 